\title{
CORRELAÇÃO ENTRE HIPÓTESE DIAGNÓSTICA E LAUDO DE TOMOGRAFIA AXIAL COMPUTADORIZADA CRANIANA
}

\author{
Edison Matos Nóvak', Fábio Terabe ${ }^{2}$, André Luís Nasimoto3, \\ Paulo Henrique Migliari de Carvalho ${ }^{4}$, Ariane Desirée Loper ${ }^{5}$, \\ Fabiana Schenato Büchele ${ }^{6}$, Júlio César Nogara7
}

\begin{abstract}
RESUMO - Para avaliar a utilização da tomografia axial computadorizada craniana (TC) em nosso meio, analisamos as solicitações de 367 exames consecutivos no período compreendido entre 07/1995 e 07/1996. Houve ampla variação da faixa etária, com média de 31,7 $\pm 22,9$ anos. As especialidades que mais solicitaram o exame foram: Neurologia (36,2\%), Pronto Atendimento $(17,4 \%)$, Neuropediatria $(16,9 \%)$ e Clínica Médica $(5,9 \%)$. As indicações mais frequentes para o exame foram: convulsão $(30 \%)$, cefaléia $(26,2 \%)$, déficit motor $(20,2 \%)$ e redução do nível de consciência $(16,9 \%)$. As "hipóteses" aventadas com maior frequência foram: afastar lesão estrutural $(9,0 \%)$, acidente vascular não discriminado $(8,2 \%)$ e neurocisticercose $(8,2 \%)$. 0 laudo da TC foi normal em $50,4 \%$ dos exames. Os laudos anormais mais frequentes foram: hidrocefalia $(5,4 \%)$, acidente vascular isquêmico $(5,4 \%)$ e neoplasia $(3,5 \%)$. As maiores taxas de achados normais foram encontradas nas "hipóteses" de cefaléia $(94,4 \%)$, convulsão $(71,4 \%)$ e afastar lesão estrutural $(66,7 \%)$. Os maiores índices de acerto entre hipótese e laudo foram encontrados nas situações de "afastar lesões estruturais" $(66,7 \%)$, hidrocefalia (50\%), acidente vascular cerebral isquêmico (50\%) e hematoma (50\%). Concluimos que a utilização de um recurso auxiliar de diagnóstico como a TC cumpre melhor ainda a sua função se o requisitante do exame preencher com clareza e detalhamento o pedido do exame.
\end{abstract}

PALAVRAS-CHAVE: tomografia computadorizada de crânio, cefaléia, lesões estruturais cerebrais.

Correlation between diagnostic hypothesis and result of cranial computed axial tomography

\begin{abstract}
The use of computed axial tomography (CT) in the investigation of neurologic disorders is attractive for his disponibility in the health services. However, the indications of the exam and the correlation with the clinical features has not been frequently studied. We study correlation between the requests of CT and the findings reported by the radiologist, in 367 exams performed from 07/1995 to 07/ 1996. The mean age was $31.7 \pm 22.9$ years. The CT were requested in decrescent order of frequency by the Services of Neurology (36.2\%), Emergency room (17.4\%), Pediatric Neurology (16.9\%) and Internal Medicine (5.9\%). The CT was more indicated in cases of seizures $(30 \%)$, headache $(26.2 \%)$, motor impairment $(20.2 \%)$ and reduction of conscience level (16.9\%). The main hypothetic diagnosis were "to discard anatomic lesions" $(9.0 \%)$, not specified stroke (8.2\%) and neurocisticercosis (8.2\%). The result of the CT was normal in $50.4 \%$ of the exams specially those requested in cases of headache $(94.4 \%)$, seizures $(71.4 \%)$ and "to discard anatomic lesions"(66.7\%).The more frequently CT abnormalities were hydrocephalus (5.4\%), ischemic stroke $(5.4 \%)$ and neoplasm (3.5\%) The greatest rates of correlation were among those to discard anatomic lesions $(66,7 \%)$, hydrocephalus (50\%), ischemic stroke $(50 \%)$ and hematoma $(50 \%)$. We concluded that CT is more helpfull if more clinical data is provided in the request form, so aiding the radiologist in the final report.
\end{abstract}

KEY WORDS: cranial computed axial tomography, headache, cranial computed tomography request.

Desde o seu desenvolvimento em 1972, a tomografia axial computadorizada (TC) é exame de amplo emprego na prática médica, sobretudo na avalia- ção de afecções que atingem a caixa craniana e o encéfalo'. Muitos estudos têm avaliado o uso das TC cranianas em diversos problemas, incluindo trau-

Estudo realizado nos Serviços de Neurologia e de Radiologia do Hospital de Clínicas da Universidade Federal do Paraná (UFPR), Curitiba PR, Brasil: ${ }^{1}$ Professor Adjunto de Neurologia da UFPR; ${ }^{2}$ Médico Residente do $2^{\circ}$ ano de Cirurgia Geral/UFPR; ${ }^{3}$ Médico Residente do $2^{\circ}$ ano de Anestesiologia/UFPR; ${ }^{4}$ Médico Residente do $2^{\circ}$ ano de Clínica Médica/UFPR; ${ }^{5}$ Médica Residente do $2^{\circ}$ ano de Psiquiatria/UNICAMP; ${ }^{6}$ Médica Generalista; ${ }^{7}$ Médico Residente do $1^{\circ}$ ano de Obstetrícia/Maternidade Santa Brígida.

Recebido 29 Novembro 2000, recebido na forma final 4 Maio 2001. Aceito 15 Maio 2001. 
matismo crânio-encefálico, acidente vascular cerebral (AVC), cefaléia e síncope ${ }^{2}$. Entretanto, ainda são controveritdos e pouco analisados os critérios de indicação da TC, para definir quando esta irá auxiliar no diagnóstico e/ou influenciar no prognóstico bem como sua relação custo-benefício em face da escassez de recursos financeiros para o setor de saúde em nosso país. Devido ao seu alto padrão de resolução, é tentadora a hipótese de usar de imediato a TC para se tentar elucidar um caso, às vezes em detrimento de meticulosa avaliação clínico-neurológica e consequente indicação dos exames complementares de acordo com o raciocínio médico. Esta conduta é aplicada mesmo em hospitais universitários, justamente naqueles em que há a formação médica de graduação e pós-graduação e onde o acesso à tomografia é menos restrito, apesar dos diversos fatores que poderiam limitar o seu uso.

Ainda não há descrição na literatura de um protocolo exato para a solicitação de TC craniana, cabendo a cada médico ou Serviço a eventual definição de seus próprios critérios de utilização. Este estudo analisou a correlação entre os achados de tomografia e a suspeita clínica prévia à sua realização, para avaliar o grau de precisão da indicação do exame. Também teve como objetivo investigar quais foram as situações clínicas para as quais a TC tem maior número de solicitações e para quais faixas etárias ela é mais utilizada. Através de abordagem das variáveis que compõem a realização de uma TC, tentou-se delinear o modo como tal exame apresenta-se inserido no contexto de um hospital universitário.

\section{MÉTODO}

Foi realizado estudo transversal em que se analisaram todas as solicitações de TC craniana, no período de julho de 1995 a julho de 1996, e os exames executados no Serviço de Radiologia do Hospital de Clínicas de Curitiba. Este Hospital é um centro de atendimento terciário vinculado à Universidade Federal do Paraná e atende cerca de 800 mil consultas por ano. Os exames tomográficos foram realizados em um dos aparelhos do Hospital, de marcas Phillips (Tomoscan 350) e Siemens (Somaton ART).

Do total de 445 exames realizados no período de um ano, foram excluídas 60 tomografias solicitadas em caráter de "controle" e 18 tomografias com dados incompletos, restando 367 exames sobre os quais realizamos este estudo.

Foram analisados os seguintes dados (a partir da requisição da TC): identificação do doente, caráter eletivo ou emergencial da solicitação, serviço de origem do doente, resumo do quadro clínico, hipótese diagnóstica e/ou objetivo do exame. Os dados clínicos foram então comparados com o laudo emitido pelos radiologistas.
Os achados da tomografia foram classificados, de acordo com as informações contidas no laudo, em normais, anormais não significativos e anormais significativos. Os achados "anormais não significativos" foram definidos como: atrofia cerebral / diminuição volumétrica de hemisfério cerebral discreta em pacientes acima de 50 anos e calcificações ${ }^{1,2}$ (localizadas em gânglios da base, plexo coróide, foice e glândula pineal), quando estas alterações não podiam ser relacionadas aos sinais e/ou sintomas do paciente.

\section{RESULTADOS}

A idade variou de menos de 1 ano até 99 anos, com média de 31,7 $\pm 22,9$ anos; em 5 requisições não constava a idade do doente (Tabela 1). Cento e oitenta e nove pacientes eram do sexo masculino $(51,5 \%)$ e os restantes 178 do feminino (48,5\%).

A distribuição da proveniência das requisições de TC está detalhada na Tabela 2, em que é bem caracterizado o predomínio dos Serviços de Neurologia, Pronto-atendimento e Neuropediatria (259 requisições ou $70,57 \%$ do total). Entre as clínicas ou serviços incluídos na denominação "outros", as que mais solicitaram exames de tomografia foram a Otorrinolaringologia $6(1,6 \%)$, Endocrinologia $5(1,4 \%), \mathrm{Ci}-$ rurgia Torácica $4(1,1 \%)$, Unidade de Terapia Intensiva $4(1,1 \%)$ e Ginecologia/Obstetrícia $3(0,8 \%)$. Cento e trinta e quatro exames $(36,5 \%)$ foram realizados em caráter emergencial e 216 (58,85\%) como exames eletivos.

As indicações clínicas mais frequentes para requisição do exame foram: convulsão 110 (30\%), cefaléia $96(26,2 \%)$, déficit motor $74(20,2 \%)$, redução do nível de consciência 62 (16,9\%), déficit sensitivo 28 (7,6\%). Várias outras indicações foram registradas, tais como atraso do desenvolvimento psicomo-

Tabela 1. Distribuição das faixas etárias dos pacientes estudados.

\begin{tabular}{ccc}
\hline Faixa etária (anos) & $N$ & $(\%)$ \\
\hline $0-9$ & 70 & $(19,0)$ \\
$10-19$ & 62 & $(16,8)$ \\
$20-29$ & 57 & $(15,5)$ \\
$30-39$ & 49 & $(13,3)$ \\
$40-49$ & 35 & $(9,5)$ \\
$50-59$ & 37 & $(10,0)$ \\
$60-69$ & 23 & $(6,2)$ \\
$70-79$ & 23 & $(6,2)$ \\
$80-89$ & 4 & $(1,0)$ \\
$90-99$ & 2 & $(0,5)$ \\
Não informado & 5 & $(1,3)$ \\
\hline
\end{tabular}


Tabela 2. Procedência e caráter das requisições de TC.

\begin{tabular}{lccccc}
\hline Clínica/Serviço & \multicolumn{5}{c}{ N de requisições } \\
& Emergência & Eletiva & Desconhecido* & N & $\%$ \\
\hline Neurologia & 22 & 106 & 5 & 133 & $(36,2)$ \\
Pronto Atendimento & 57 & 4 & 3 & 64 & $(17,4)$ \\
Neuropediatria & 8 & 51 & 3 & 62 & $(16.9)$ \\
Clínica Médica & 9 & 12 & 1 & 22 & $(6,0)$ \\
Neurocirurgia & 4 & 10 & 1 & 15 & $(4,0)$ \\
Infectologia & 7 & 5 & - & 12 & $(3,2)$ \\
Hematologia & 8 & 3 & - & 11 & $(3,0)$ \\
Emergências Clínicas & 7 & 2 & 1 & 10 & $(2,7)$ \\
Outros & 12 & 23 & 3 & 38 & $(10,3)$ \\
Total & 134 & 216 & 17 & 367 & \\
\hline
\end{tabular}

*Não foi possível determinar o caráter da TC.

tor $(n=22)$, náuseas/vômitos $(n=14)$, traumatismo crânio-encefálico $(n=12)$, tontura $(n=11)$ e alteração de conduta $(n=7)$, entre outras. Notou-se também a presença de mais de 1 indicação em diversas requisições (Tabela 3 ).

As hipóteses diagnósticas mais frequentes formuladas pelo médico que solicitou a TC estão relacionadas na Tabela 4. Cabe citar que, na requisição padronizada no Hospital de Clínicas, separa-se "indicação clínica" de "hipótese diagnóstica".Uma grande variedade de outras hipóteses foi encontrada neste levantamento, inclusive abrangendo o maior número de requisições de exame (75 ou 20,4\%). Entre estas, podemos citar: lesão hipóxico-isquêmica $(n=7)$, atraso do desenvolvimento psicomotor $(n=5)$, lesão expansiva $(n=4)$ e demência $(n=2)$. Propositadamente relacionamos o termo exato usado no item hipótese, não agrupando, para fins de análise, situações com alguma equivalência clínica.

A frequência de laudos emitidos pelo Serviço de Radiologia, em ordem decrescente, foi: normal 185 (50,4\%), calcificações não-cisticercóticas $37(10,0 \%)$, diminuição volumétrica 35 (9,5\%), hidrocefalia 20 $(5,4 \%)$, AVC isquêmico $20(5,4 \%)$, neurocisticercose $15(4,0 \%)$, neoplasia $13(3,5 \%)$, área de hipodensidade $12(3,2 \%)$, AVC hemorrágico $8(2,2 \%)$, hematoma extradural ou subdural $8(2,2 \%)$ e outros $30(8,1 \%)$. Os "outros"achados de tomografia foram principalmente alterações vasculares (sinais de doença de pequenos vasos) 11 , lesões expansivas não caracterizadas como neoplasia 7, cistos aracnoideos 4 e edema cerebral 3 (Tabela 5).

A relação dos achados tomográficos em relação à faixa etária dos pacientes é demonstrada na Tabela 6 . Em alguns casos ocorreu mais de uma anorma-
Tabela 3. Indicações para realização do exame.

\begin{tabular}{lcc}
\hline Indicação & $\mathrm{N}$ & $(\%)$ \\
\hline Convulsão & 110 & $(30)$ \\
Cefaléia & 96 & $(26,2)$ \\
"Déficit motor" & 74 & $(20,2)$ \\
Redução do nível de consciência & 62 & $(16,9)$ \\
"Déficit sensitivo" & 28 & $(7,6)$ \\
Outras & 66 & \\
Total & 436 & \\
\hline
\end{tabular}

${ }^{*} \mathrm{O}$ número total de indicações é superior ao de exames analisados pois ocorreu mais de 1 citação em alguns casos.

Tabela 4. Correlação entre as hipóteses diagnósticas e/ ou objetivo do exame com o número de pacientes.

\begin{tabular}{lcc}
\hline Hipótese & $\mathrm{N}$ & $(\%)$ \\
\hline Afastar lesão estrutural & 33 & $(9,0)$ \\
AVC não discriminado & 30 & $(8,2)$ \\
Neurocisticercose & 30 & $(8,2)$ \\
Epilepsia & 26 & $(7,0)$ \\
Neoplasia & 24 & $(6,5)$ \\
Hidrocefalia & 22 & $(6,0)$ \\
AVC isquêmico & 22 & $(6,0)$ \\
Convulsão & 21 & $(5,7)$ \\
Traumatismo crânio encefálico & 21 & $(5,7)$ \\
Cefaléia & 18 & $(4,9)$ \\
AVC hemorrágico & 13 & $(3,5)$ \\
Hipertensão intracraniana & 12 & $(3,2)$ \\
Hematoma & 12 & $(3,3)$ \\
Metástase & 8 & $(2,2)$ \\
Outras & 75 & $(20,4)$ \\
\hline
\end{tabular}


Tabela 5. Características da redação dos laudos e sua frequência em relação ao número total de casos estudados e ao total de laudos com anormalidades*.

\begin{tabular}{lccc}
\hline Laudo & $\mathrm{N}$ & $\begin{array}{c}\text { \% em relação } \\
\text { ao total de casos }\end{array}$ & $\begin{array}{c}\text { \% em relação } \\
\text { ao total de alterações }\end{array}$ \\
\hline Normal & 185 & 50,4 & 48,3 \\
Calcificações não-cisticercóticas & 37 & 10,0 & 9,6 \\
Diminuição volumétrica & 35 & 9,5 & 9,1 \\
Hidrocefalia & 20 & 5,4 & 5,2 \\
AVC isquêmico & 20 & 5,4 & 5,2 \\
Neurocisticercose & 15 & 4,0 & 3,9 \\
Neoplasia & 13 & 3,5 & 3,4 \\
Área de hipodensidade & 12 & 3,2 & 3,1 \\
AVC hemorrágico & 8 & 2,2 & 2,0 \\
Hematoma extra ou subdural & 8 & 2,2 & 2,0 \\
Outros & 30 & 8,1 & 7,8 \\
\hline
\end{tabular}

*Em alguns casos foi citada mais de uma alteração no laudo.

lidade registrada no laudo e todas elas foram consideradas na análise, ultrapassando-se assim o número total de exames analisados.

A relação entre as hipóteses aventadas e o achado de exames normais se encontra na Figura 1. Houve maior percentual de laudos com "normalidade" quando as hipóteses/objetivo do exame foram: cefaléia $(94,4 \%)$, convulsão $(71,4 \%)$, neoplasia $(68,3 \%)$, afastar lesão estrutural $(66,7 \%)$ e traumatismo crânioencefálico $(63,7 \%)$. Registrou-se que as maiores percentagens de correspondência exata entre o laudo e as hipóteses ou objetivo do exame foram nas situa- ções de afastar lesão estrutural $(66,7 \%)$, AVC isquêmico $(50,0 \%)$, hidrocefalia $(50,0 \%)$ e hematoma extra ou subdural $(50,0 \%)$.

$\mathrm{Na}$ análise geral, os achados tomográficos foram classificados em: normais em 185 pacientes $(50,4 \%)$, anormais significativos em $149(40,6 \%)$ e anormais não significativos em 33 (9,0\%). Esta distribuição, de acordo com as hipóteses mais frequentes, está descrita na Tabela 7. Não foram considerados os laudos daqueles exames em que havia hipóteses ou o motivo do exame apresentava baixa frequência ou este não era explicitado.

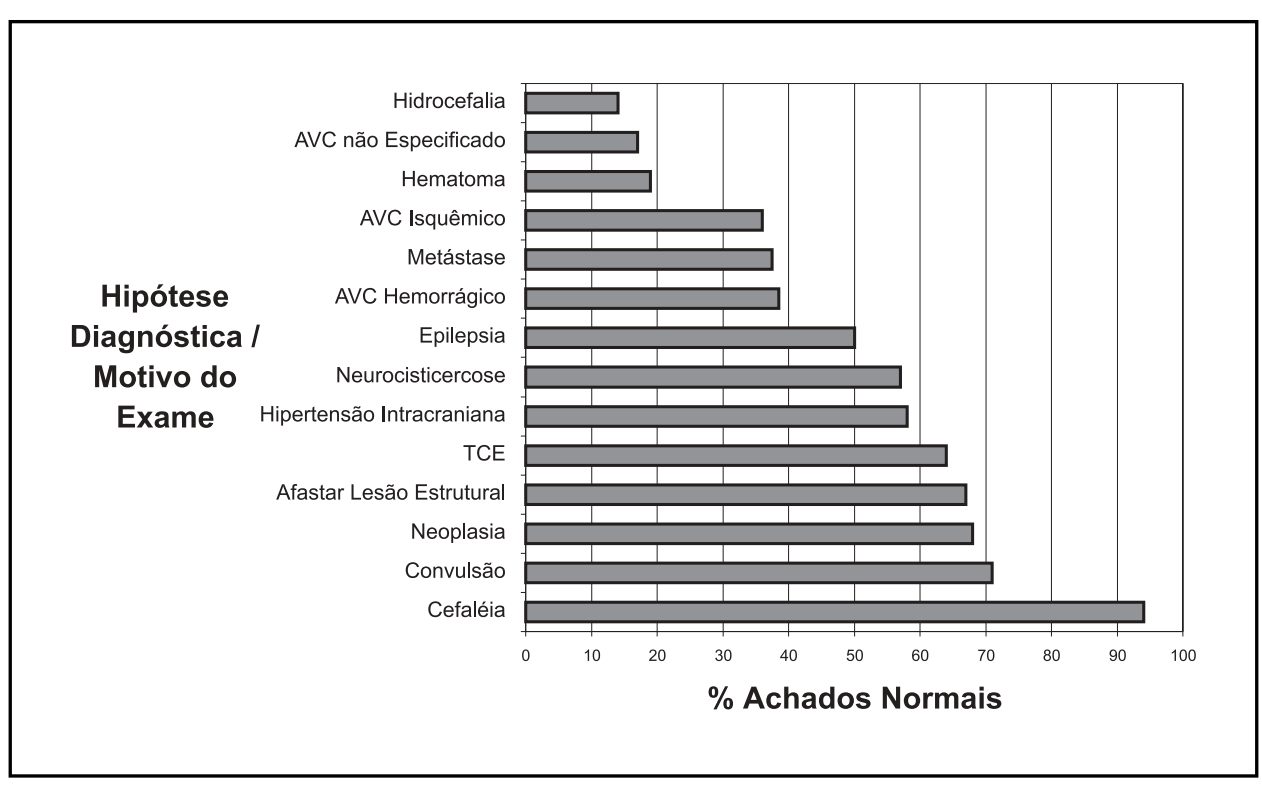

Fig 1. Frequência de laudos normais em relação à hipótese diagnóstica ou motivo do exame. 
Tabela 6. Correlação entre os resultados dos laudos e sua distribuição nas diversas faixas etárias*.

\begin{tabular}{|c|c|c|c|c|}
\hline Laudo & $\begin{aligned} & N(\%) \\
< & 15 \text { anos }\end{aligned}$ & $\begin{array}{c}\mathrm{N}(\%) \\
15-39 \text { anos }\end{array}$ & $\begin{array}{c}N(\%) \\
40-59 \text { anos }\end{array}$ & $\begin{aligned} & \text { N (\%) } \\
> & 59 \text { anos }\end{aligned}$ \\
\hline Normal & $63(58,3)$ & $75(55,5)$ & $30(40,0)$ & $17(26,1)$ \\
\hline Hidrocefalia & $11(10,2)$ & $5(3,7)$ & $4(5,3)$ & - \\
\hline AVC isquêmico & $1(0,9)$ & $4(3,0)$ & $7(9,3)$ & $8(12,3)$ \\
\hline Neoplasia & $6(5,6)$ & $2(1,5)$ & $3(4,0)$ & $2(3,1)$ \\
\hline AVC hemorrágico & $2(1,8)$ & $1(0,7)$ & $4(5,6)$ & $1(1,5)$ \\
\hline Hematoma extra ou subdural & $2(1,8)$ & - & $3(4,0)$ & $3(4,6)$ \\
\hline Edema cerebral & - & $2(1,5)$ & $1(1,3)$ & - \\
\hline Neurocisticercose & $4(3,7)$ & $7(5,2)$ & $4(5,3)$ & - \\
\hline Diminuição volumétrica & $4(3,7)$ & $5(3,7)$ & $7(9,3)$ & $19(29,2)$ \\
\hline Calcificações & $7(6,5)$ & $16(11,8)$ & $10(13,3)$ & $4(6,1)$ \\
\hline Lesão expansiva & $2(1,8)$ & $2(1,5)$ & - & $3(4,6)$ \\
\hline Cisto aracnóide & $2(1,8)$ & $2(1,5)$ & - & - \\
\hline Área hipodensa & $2(1,8)$ & $6(4,4)$ & $2(2,6)$ & $2(3,1)$ \\
\hline Metástase & - & $1(0,7)$ & - & - \\
\hline Doença de pequenos vasos & - & - & - & $6(9,2)$ \\
\hline Outros & $2(1,8)$ & $7(5,2)$ & - & - \\
\hline Total & 108 & 135 & 75 & 65 \\
\hline
\end{tabular}

Tabela 7. Distribuição dos achados de acordo com a hipótese.

\begin{tabular}{|c|c|c|c|c|c|}
\hline \multirow[t]{3}{*}{ Hipótese/objetivo do exame } & \multirow[t]{3}{*}{$\mathrm{N}$} & \multirow[t]{3}{*}{ Normal (\%) } & \multicolumn{3}{|c|}{ Anormal (\%) } \\
\hline & & & \multicolumn{2}{|c|}{ Significativo } & \multirow[t]{2}{*}{ Não significativo } \\
\hline & & & Compatível & Incompatível & \\
\hline Afastar lesão estrutural & 33 & $22(66,7)$ & $5(15,1)$ & - & $6(18,2)$ \\
\hline AVC não discriminado & 30 & $5(16,7)$ & $13(43,3)$ & $4(13,3)$ & $8(26,7)$ \\
\hline Neurocisticercose & 30 & $17(56,7)$ & $7(23,3)$ & $1(3,3)$ & $5(16,6)$ \\
\hline Epilepsia & 26 & $13(50)$ & $3(11,6)$ & - & $10(38,4)$ \\
\hline Neoplasia & 24 & $14(58,3)$ & $7(29,2)$ & - & $3(12,5)$ \\
\hline AVC isquêmico & 22 & $8(36,4)$ & $11(50)$ & $1(4,5)$ & $2(9)$ \\
\hline Hidrocefalia & 22 & $3(13,6)$ & $11(50)$ & $3(13,5)$ & $5(22,5)$ \\
\hline Traumatismo crânio encefálico & 21 & $14(66,7)$ & $2(9,6)$ & $2(9,6)$ & $3(14,3)$ \\
\hline Cefaléia & 18 & $17(94,4)$ & - & - & $1(5,6)$ \\
\hline AVC hemorrágico & 13 & $5(38,5)$ & $4(30,8)$ & $2(15,4)$ & $2(15,4)$ \\
\hline Hipertensão intracraniana & 12 & $7(58,3)$ & $2(16,7)$ & $2(16,7)$ & $1(8,3)$ \\
\hline Hematoma & 12 & $2(16,7)$ & $8(66,6)$ & $2(16,7)$ & - \\
\hline Metástase & 8 & $3(37,5)$ & $1(12,5)$ & - & $4(50)$ \\
\hline
\end{tabular}


Tabela 8. Percentual de normalidade por hipótese diagnóstica na lieratura.

\begin{tabular}{lcccc}
\hline Hipótese & \multicolumn{4}{c}{ Autor } \\
\cline { 2 - 5 } & Mills et col. & Reinus et col. & Reinus et col. & presente amostra \\
\hline "Cefaléia" & 71,0 & 65,7 & 91,3 & 94,4 \\
"Convulsão" & 71,0 & 30,4 & 80,3 & 71,4 \\
Trauma & 61,0 & 61,2 & 87,5 & 63,7 \\
\hline
\end{tabular}

\section{DISCUSSÃO}

A população estudada abrangeu ampla faixa etária, estendendo-se desde o período neonatal até à senilidade. Houve mais solicitações de exames nas faixas etárias menores (29,4\% com menos de 15 anos) que em idosos (14,2\% tinham mais de 60 anos).

As solicitações de caráter eletivo responderam por $63,5 \%$ dos casos, denotando o maior uso deste recurso diagnóstico pelo serviços ambulatoriais do Hospital de Clínicas.Esta constatação deve-se ao fato de que este Hospital possui maior demanda para atendimento ambulatorial de nível terciário do que de emergência (no período em que foram coletados os dados, o número de consultas emergenciais foi de aproximadamente 100 mil e o de consultas ambulatoriais cerca de $384 \mathrm{mil}$ ). A especialidade com maior número de pedidos foi a Neurologia, com $133 \mathrm{ca}$ sos, seguida pelo Pronto-Atendimento com $64 \mathrm{ca}-$ sos (este, obviamente, com maior proporção de solicitações emergenciais de exames) e pela Neuropediatria, com 62 casos.

O dado clínico mais citado foi convulsão (30,0\%), seguido de cefaléia $(26,2 \%)$, déficit motor $(20,2 \%)$ e redução do nível ou perda de consciência $(16,9 \%)$. Como parâmetro, podemos citar dados de um artigo publicado por Reinus e col. ${ }^{3}$, que encontraram como variáveis clínicas mais freqüentes a história de trauma $(37,9 \%)$, cefaléia $(34,5 \%)$, perda de consciência $(21,8 \%)$ e déficit focal $(18,3 \%)$.

As suspeitas clínicas ou os objetivos do exame mais citados foram, em ordem decrescente de aparecimento: AVC, neurocisticercose, neoplasias e hidrocefalia. Em contrapartida, estudo realizado em um serviço de emergência demonstrou as seguintes indicações de realização de TC (excluído o trauma que perfaz $49 \%)$ : cefaléia $(25,1 \%)$, convulsões $(15,1 \%)$, AVC (14,2\%) e 13,7\% outras razões ${ }^{4}$. Pôde-se observar que algumas solicitações traziam, no item "objetivo do exame", um sintoma e não uma hipótese diagnóstica, como no caso de "convulsão" (21 casos) e "cefaléia" (18 casos). Isto poderia refletir má elaboração da hipótese diagnóstica ou apenas mau pre- enchimento das requisições, fato que ocorre também em outros Serviços, como já foi descrito em estudos semelhantes ${ }^{4}$. Outros exames tinham como hipótese diagnóstica "afastar lesão estrutural" (33 casos), termo que não esclarece o motivo da solicitação, dificultando a interpretação do radiologista. Algumas solicitações referiam como objetivo do exame apenas "crânio" ou "investigação" e em outras não foi preenchido este campo do pedido de tomografia. Na coleta dos dados constatou-se falhas no preenchimento das solicitações como: ausência de dados, história clínica ou exame físico incompletos, abreviações indecifráveis e grafia ruim, o que também cria entraves à elaboração diagnóstica do radiologista. Estes fatores trouxeram dificuldades significativas, tanto pela exclusão de exames como pela impossibilidade de analisar detalhadamente os graus de correlação entre a história clínica, a hipótese diagnóstica e o laudo tomográfico.

A ocorrência de exames dentro da normalidade em pouco mais da metade dos indivíduos desta amostra (50,4\%), embora pareça elevada, pode ser comparada a alguns estudos realizados em hospitais ligados a programas de Residência Médica que demonstraram taxas de $40,3 \%, 53,9 \%$ e até $84,3 \%^{3-6}$.

Esta taxa de achados normais não teve distribuição homogênea nos diferentes grupos de hipóteses, como demonstrado na Figura 1. Pôde-se observar que os achados de normalidade foram mais significativos entre as hipóteses de cefaléia, convulsão e "afastar lesão estrutural". Em relação à cefaléia, os resultados apresentam coerência com a literatura, visto que estudos demonstraram taxa de exames normais variando de $88,4 \%$ a $92 \%$ em pacientes com cefaléia sem outros sinais neurológicos, mesmo após uso de contraste ${ }^{2,6}$. No que concerne à hipótese "convulsão", a proporção de exames normais descrita chega a $72 \%$, equivalente àquela encontrada nesta amostra ${ }^{7}$. Estas relações estão referidas na Tabela 8.

Deve-se salientar que um laudo de " normal" não indica necessariamente desacerto na indicação da tomografia, pois este exame complementar deve fa- 
zer parte da investigação de muitas suspeitas etiológicas inclusive, e especialmente naquelas em que há pobreza ou ausência de dados clínicos. A maior taxa de acerto entre hipótese e laudo foi encontrada nos itens "afastar lesão estrutural" (em que o achado normal foi considerado "correspondente"), hidrocefalia, AVC isquêmico e hematoma extra ou subdural. As taxas de acerto entre hipótese e laudo encontradas por Reinus e col. ${ }^{5}$ foram de $50 \%$ para AVC hemorrágico, $75,4 \%$ para AVC isquêmico e $60 \%$ para neoplasia. Como o critério para elaboração do gráfico foi a correlação exata entre hipótese e laudo, as hipóteses de cefaléia, convulsão, epilepsia e TCE não tiveram correspondência alguma. Entre os achados que não correspondiam à hipótese, havia vários resultados significativos, tais como 4 casos de AVCs isquêmicos, 4 de hidrocefalias, 3 de neoplasias e 3 de AVCs hemorrágicos. $O$ mesmo estudo ${ }^{5}$ também relata o encontro de achados clinicamente significantes em 17 de 536 exames realizados.

Em relação à faixa etária, os achados mais freqüentes foram os seguintes (Tabela 5): (a) abaixo de 15 anos: hidrocefalia e calcificações; (b) entre 15 e 39 anos: calcificações e neurocisticercose; (c) entre 40 e 59 anos: calcificações, diminuição volumétrica e AVC isquêmico; (d) acima de 60 anos: diminuição volumétrica e AVC isquêmico. Considerando-se apenas os achados significativos, os laudos mais encontrados em relação à idade foram: (a) abaixo de 15 anos: hidrocefalia (9 casos), neoplasia (6 casos) e neurocisticercose (3 casos); (b) entre 15 e 39 anos: neurocisticercose (7 casos), hidrocefalia (5 casos) e AVC isquêmico (4 casos); (c) entre 40 e 59 anos: AVC isquêmico (7 casos), hidrocefalia (4 casos), neurocisticercose (4 casos) e AVC hemorrágico (4 casos); (d) acima de 60 anos: AVC isquêmico (8 casos), hematoma (3 casos) e neoplasia ( 2 casos).

Podemos, portanto, constatar que a maioria dos achados da presente análise apresentou certa coerência em relação aos dados disponíveis na literatura. O elevado número de hipóteses de hidrocefalia pode ser justificado pela grande proporção de crianças e adultos jovens na amostra, decorrente da fonte de encaminhamento dos pedidos. Da mesma forma, explica-se a taxa de hipóteses de neurocisticer- cose pela alta prevalência de tal doença em nossa região. Em relação aos achados das TC de crânio, deve ser realçado apenas o grande número de calcificações encontrado em quase todas as faixas etárias e o predomínio de AVCs isquêmicos nos indivíduos idosos, o que é esperado face às alterações degenerativas vasculares esperadas neste grupo de pacientes.

Embora haja grande número de tomografias com laudo de normalidade, não se pôde concluir, com base nestes dados e nas referências pesquisadas, qual a proporção de exames que são indevidamente solicitados, mesmo que por excesso de zelo por parte do médico atendente. De fato, não há possibilidade real de analisar tal dado em estudo retrospectivo, pois a indicação deste exame complementar depende em grande parte do quadro clínico apresentado pelo doente por ocasião do primeiro atendimento, bem como da experiência do médico atendente e sua capacidade de elaborar hipóteses diagnósticas mais objetivas. Este estudo demonstrou a importância da conscientização dos médicos para que se proceda ao correto preenchimento dos formulários de solicitação de TCs cranianas, visto que dele depende melhor interpretação do exame pelo radiologista e maior benefício ao paciente. Ao mesmo tempo, parece ser indicada uma avaliação crítica e modificações nos formulários de requisição do exame, sempre com o objetivo de estimular o adequado preenchimento do pedido a partir, também, de uma adequada e pormenorizada avaliação clínica.

\section{REFERÊNCIAS}

1. Lee SH, Kieffer AS, Montoya JH. Pitfalls and limitations of CT in neurodiagnosis. In Lee SH, Rao KCVG (eds). Cranial computed tomography. New York: McGraw-Hill 1983;681-699.

2. Demaarel P, Boelaert I, Wilms G, et al. The role of cranial computed tomography in the diagnostic work-up of headache. Headache 1996; 36:347-348

3. Reinus WR, Erickson KK, Wippold II FJ. Unenhanced emergency cranial CT: optimizing patient selection with univariate and multivariate analyses. Radiology 1993;186:763-768.

4. Alfaro D, Lewitt MA, English DK, et al. Accuracy of interpretation of cranial computed tomography scans in an emergency medicine residency program. Ann Intern Med 1995;25:169-174.

5. Reinus WR, Zwemer FL. Clinical prediction of emergency cranial computed tomography results. Ann Intern Med 1994;23:1271-1278.

6. Akpek S, Arac M, Atilla S, et al. Cost-effectiveness of computed tomography in the evaluation of patients with headache. Headache 1995; 35:228-230.

7. Mills ML, Russo LS, Vines FS, et al. High-yield criteria for urgent cranial computed tomography scans. Ann Emerg Med 1986;15:1167-1172. 